\title{
Prosthetic Ring Endocarditis with Shrunken Mitral Annulus Late After Undersized Mitral Annuloplasty
}

\author{
Masaki Hamamoto*, Taira Kobayashi, Masamichi Ozawa, Hiroshi Kodama \\ Department of Cardiovascular Surgery, JA Hiroshima General Hospital, Hiroshima, Japan \\ Email: ${ }^{*}$ cardiovascsurgeon@gmail.com
}

Received 10 June 2014; revised 10 July 2014; accepted 10 August 2014

Copyright $@ 2014$ by authors and Scientific Research Publishing Inc.

This work is licensed under the Creative Commons Attribution International License (CC BY). http://creativecommons.org/licenses/by/4.0/

\section{cC) (i) Open Access}

\begin{abstract}
We report a case of supra-annular mitral valve replacement for a patient with prosthetic ring endocarditis that developed late after undergoing undersized mitral annuloplasty (UMAP). A 58year-old man had undergone left ventriculoplasty and UMAP with a 26-mm semi-rigid full ring for dilated cardiomyopathy and functional mitral regurgitation. The mitral valve had progressively stenosed and he developed prosthetic ring endocarditis 5 years after the operation. In the surgery, there were brownish fresh vegetations around the anterior mitral leaflet and the prosthetic ring. The mitral annulus was very small, even after removal of the infected ring prosthesis and debridement of the infected tissues. We implanted a $22-\mathrm{mm}$ mechanical prosthesis in the supra-annular position using everting mattress sutures to prevent the valve leaflets from getting stuck by the thickened subannular tissue. Herein, we discuss the surgical procedures for a shrunken mitral annulus.
\end{abstract}

\section{Keywords}

Undersized Mitral Annuloplasty, Shrunken Mitral Annulus, Supra-Annular Mitral Valve Replacement

\section{Introduction}

Undersized mitral annuloplasty (UMAP) is one of the surgical techniques applied for functional mitral regurgitation (MR) in dilated or ischemic cardiomyopathy [1]. There are several complications of this procedure, including recurrent MR, functional mitral stenosis [2] and prosthetic ring endocarditis [3]. While these complica-

*Corresponding author.

How to cite this paper: Hamamoto, M., Kobayashi, T., Ozawa, M. and Kodama, H. (2014) Prosthetic Ring Endocarditis with Shrunken Mitral Annulus Late After Undersized Mitral Annuloplasty. Open Journal of Thoracic Surgery, 4, 62-65. 
tions are less frequent, most patients with these complications are more likely to require reoperation. Herein, we report our experience of mitral valve replacement (MVR) for a patient with prosthetic ring endocarditis, who had a small mitral annulus affected by a prior UMAP using a 2 size-reduced semi-rigid ring.

\section{Case}

A 58-year-old man had undergone overlapping left ventriculoplasty with UMAP using a 26-mm Physio ring ${ }^{\circledR}$ (Edwards Lifesciences, Irvine, CA, USA) for dilated cardiomyopathy with functional MR. Echocardiography revealed mild MR with a mitral valve area (MVA) of $3.6 \mathrm{~cm}^{2}$ and a left atrial dimension (LAD) of $40 \mathrm{~mm}$ after the operation. He was doing well without heart failure. Four years later, the MVA decreased to $1.2 \mathrm{~cm}^{2}$ with an increment in LAD of $48 \mathrm{~mm}$ without symptoms. Five years after the operation, he developed stroke in the middle cerebral artery territory with right hemiparesis, facial nerve palsy and aphasia. Transthoracic and transesophageal echocardiography revealed an irregular-shaped mass, which was $15 \mathrm{~mm}$ in diameter, attached to the anterior mitral leaflet near the prosthetic ring. Opening of the mitral leaflets were restricted with a MVA of 0.95 $\mathrm{cm}^{2}$ and a mean transmitral pressure gradient of $9 \mathrm{mmHg}$ at rest, showing greater than moderate level of mitral stenosis. Moderate MR was also recognized (Figure 1(A)-(C)). Laboratory data showed increased inflammatory reaction with clinical signs of chill and high fever. Blood culture revealed Staphylococcus saccharolyticus. Prosthetic ring endocarditis with mitral stenosis and regurgitation were diagnosed, and surgical intervention was planned.

Through median re-sternotomy, cardiopulmonary bypass was established with bicaval and ascending aorta cannulation. The mitral valve was exposed through right-side left atriotomy. There were brownish fragile vegetations attached to the anterior mitral leaflet (Figure 1(D)) and pus formation around the semi-rigid ring (Figure $1(E))$. We carefully removed the vegetations and explanted the entire semi-rigid ring. The anterior mitral leaflet was also excised because it was completely thickened with partial inflammatory changes. The posterior mitral leaflet, which was not affected by the infection, was preserved. The 22-mm valve sizer passed tightly through the mitral annulus. Therefore, a 22-mm ATS M360 ${ }^{\mathrm{TM}}$ mechanical prosthesis (ATS medical, Inc., Minneapolis, MN, USA) was implanted in the supra-annular position using noneverting mattress sutures. However, the valve leaflets got stuck due to protrusion of the subannular fibrous tissues. We removed the prosthesis, trimmed the thickened fibrous tissue, and implanted the same-sized prosthesis in the supra-annular position using everting mattress sutures. The postoperative recovery was uneventful. Postoperative echocardiography showed effective orifice area was $2.15 \mathrm{~cm}^{2}$ and mean transprosthetic gradient was $5.3 \mathrm{mmHg}$ with no stuck valve leaflets.

The patient has continued to take oral anticoagulant (warfarin) and has been strictly followed in the outpatient clinic. He has been doing well without any signs of heart failure.

\section{Discussion}

The size of a prosthetic ring for UMAP is generally two sizes under the one applied for the degenerative MR [1]. An undersized prosthetic ring extensively reduces the anteroposterior distance of the mitral annulus, which makes a sufficient coaptation. However, long-term fixation by the prosthetic ring made the mitral annulus more rigid and inflexible. The mitral annular area would stay shrunken even after removal of the prosthetic ring. In our case, a 26-mm Physio ring ${ }^{\circledR}$ was implanted during the initial operation. The ring area of 26-mm Physio ring ${ }^{\circledR}$ is $325 \mathrm{~mm}^{2}$ as indicated by the manufacturer. The calculated diameter after circularization based on this area is about $20.3 \mathrm{~mm}$, according to the circle formula: diameter $=2 \times \sqrt{(\text { ring area } / \pi)}$. We assumed that this diameter was comparable to the mitral annular diameter. We also needed to consider patient-prosthetic mismatch (PPM), which can occur in patients with a small mitral annulus after undersized ring annuloplasty. PPM at the mitral position is defined to be less than $1.2 \mathrm{~cm}^{2} / \mathrm{m}^{2}$ [4]. In our patient with a body surface area of $1.8 \mathrm{~m}^{2}$, the orifice area of the mitral mechanical prosthesis should be greater than $2.16 \mathrm{~cm}^{2}$ to avoid PPM. Based on these figures regarding the mitral annular diameter and valve area, we selected a 22-mm mechanical valve (ATS M360 ${ }^{\mathrm{TM}}$ ), which is designed for supra-annular implantation and has a larger valve area of $3.17 \mathrm{~cm}^{2}$ comparable to that of a 25-mm standard type.

In patients with a small mitral annulus, several implanting techniques have been proposed to avoid PPM, including supra-annular valve implantation [5], aortomitral patch augmentation (Manouguian method) and composite graft replacement, termed the chimney technique [6].

Supra-annular valve implantation is the option that was selected in our case. Supra-annular MVR has been 

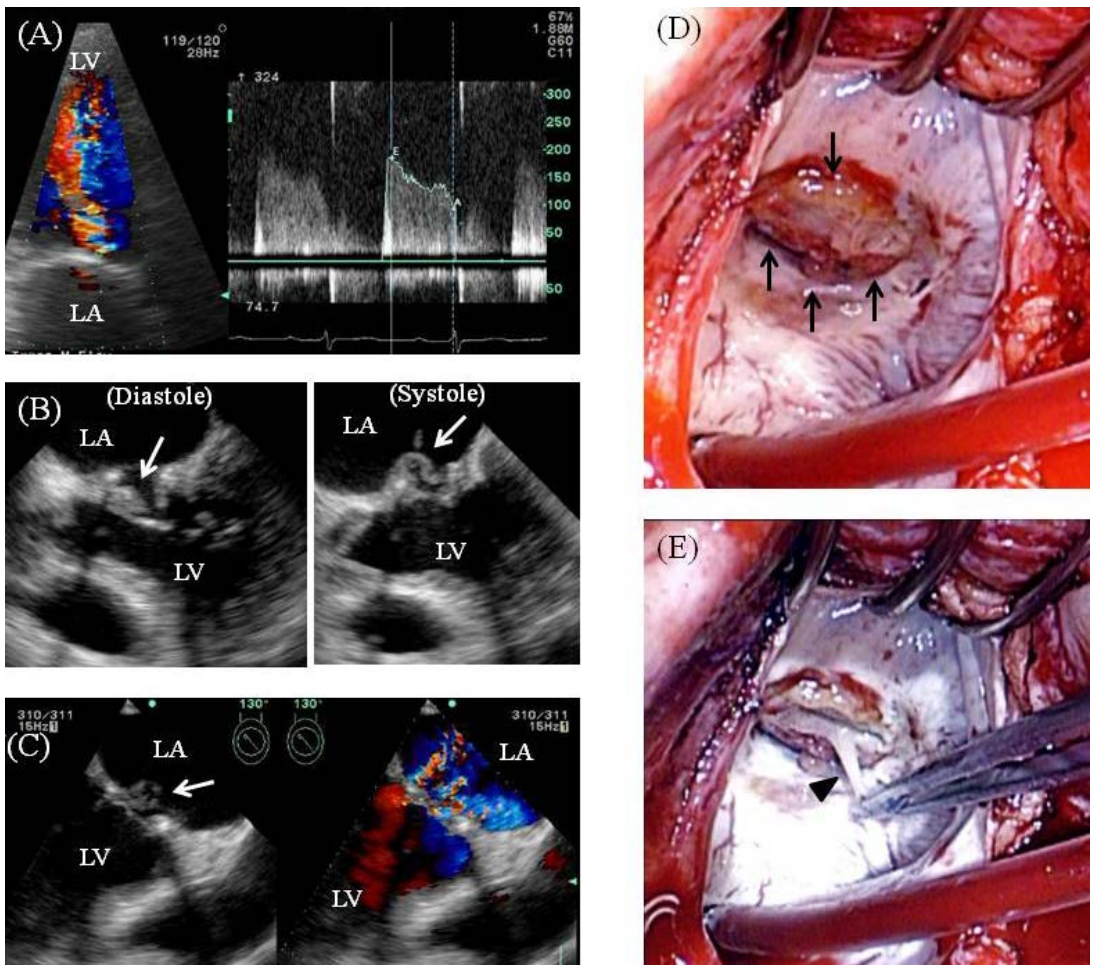

Figure 1. (A) Preoperative transthoracic echocardiography demonstrated an accelerated mitral flow velocity (the $\mathrm{E}$ wave velocity was increased to be $1.85 \mathrm{~m} / \mathrm{s}$ ) and a decreased mitral valve area of $0.95 \mathrm{~cm}^{2}$ (deceleration time was $788 \mathrm{~ms}$ ) with a mean transmitral pressure gradient of $9 \mathrm{mmHg}$, showing greater than moderate level of the mitral stenosis. (B) and (C) Preoperative transesophageal echocardiography revealed that the mobile vegetations (white arrows) were attached to the anterior mitral annulus and leaflet. The opening of the mitral leaflets was restricted with significant mitral regurgitation. LA: left atrium; LV: left ventricle. (D) and (E) Intraoperative photos of the mitral valve exposed through a right-side left atriotomy (Surgeon's view). (D) The fragile vegetation (black arrows) was attached to the anterior mitral annulus and leaflet. (E) During debridement of the vegetations and the infected tissues, purulent discharge (black arrowhead) was recognized.

performed to compensate for the prosthesis-annulus size mismatch in pediatric patients with a small mitral annulus. Kanter et al. reported that supra-annular MVR was useful for children with good operative survival. They also mentioned that this procedure had several complications, such as the coronary ischemia caused by compressing the circumflex artery by a prosthesis and the supraventricular arrhythmia including heart block requiring a permanent pacemaker [5]. In adult patients, supra-annular MVR has been applied to those with extensive calcified mitral annulus [7]. There have been few reports on this procedure attempted on the patients with narrowed mitral annulus caused by UMAP as shown in our case. Regarding the implanting technique of a mechanical prosthesis in the supra-annular position, there is a pitfall of placing the stitches on the small mitral annulus. In our patient, a mechanical prosthesis was initially implanted using noneverting mattress sutures. However, this implanting technique caused restricted leaflet motion because the protrusion of the subannular fibrous tissues interfered with the valve leaflets. We, then, applied everting mattress sutures and successfully implanted the same prosthesis in the supra-annular position. We recommend everting mattress sutures, even in cases requiring supra-annular positioning of the prosthesis for a small annulus.

Aortomitral patch augmentation according to the Manouguian method is an alternative to obtain a wider annulus and allow implantation of a larger prosthesis. This procedure is indicated for patients with concomitant aortic valve diseases or those having aortic prostheses. Jonas et al. reported a modified procedure for mitral annular patch enlargement with preservation of the aortic valve [8]. The authors enlarged the subaortic curtain and mitral annulus using a triangular patch and implanted an adequate-sized prosthesis. In our case, we decided to 
not perform such a complicated procedure because there were no aortic valve diseases and an adequate-sized prosthesis could be implanted supra-annually.

Rocafort et al. reported a novel technique using a composite graft called the "chimney technique" [6]. The chimney composite graft with a mechanical prosthesis mounted on a Dacron graft was sutured to the mitral annulus. The height of the composite graft was tailored according to the subvalvular protrusion of the prosthesis. This technique has the advantage of implanting a larger prosthesis with smooth leaflet opening within the graft without getting stuck by subvalvular fibrous tissue. This procedure may be promising when supra-annular MVR is unfeasible.

\section{Conclusion}

Supra-annular MVR using everting mattress sutures was a good option for the shrunken and rigid mitral annulus affected by a prior UMAP using a 2 size-reduced semi-rigid ring.

\section{Conflict of Interest Statement}

The authors have no conflicts of interest.

\section{References}

[1] Bolling, S.F., Deeb, G.M., Brunsting, L.A. and Bacg, D.S. (1995) Early Outcome of Mitral Valve Reconstruction in Patients with End-Stage Cardiomyopathy. The Journal of Thoracic and Cardiovascular Surgery, 109, 676-682. http://dx.doi.org/10.1016/S0022-5223(95)70348-9

[2] Magne, J., Senechal, M., Mathieu, P., Dumesnil, J.G., Dagenais, F. and Pibarot, P. (2008) Restrictive Annuloplasty for Ischemic Mitral Regurgitation May Induce Functional Mitral Stenosis. Journal of the American College of Cardiology, 51, 1692-1701. http://dx.doi.org/10.1016/j.jacc.2007.11.082

[3] Karavas, A.N., Filsoufi, F., Mihaljevic, T., Aranki, S.F., Cohn, L.H. and Byrne, J.G. (2002) Risk Factors and Management of Endocarditis after Mitral Valve Repair. Journal of Heart Valve Disease, 11, 660-664.

[4] Magne, J., Mathieu, P., Dumesnil, J.G., Tanné, D., Dagenais, F., Doyle, D. and Pibarot, P. (2007) Impact of Prosthesis-Patient Mismatch on Survival after Mitral Valve Replacement. Circulation, 115, 1417-1425. http://dx.doi.org/10.1161/CIRCULATIONAHA.106.631549

[5] Kanter, K.R., Kogon, B.E. and Kirshbom, P.M. (2011) Supra-Annular Mitral Valve Replacement in Children. The Annals of Thoracic Surgery, 92, 2221-2229. http://dx.doi.org/10.1016/j.athoracsur.2011.06.023

[6] Rocafort, A.G., Aroca, A., Polo, L., Rey, J. and Villagra, F. (2013) Chimney Technique for Mitral Valve Replacement in Children. The Annals of Thoracic Surgery, 96, 1885-1887. http://dx.doi.org/10.1016/j.athoracsur.2013.04.092

[7] Kato, Y., Hattori, K., Bito, Y., Kotani, S., Inoue, K. and Shibata, T. (2011) Simple Supra-Annular Prosthesis Insertion for Dialysis Patients with Extensive Mitral Annular Calcification. The Journal of Heart Valve Disease, 20, 180-183.

[8] Jonas, R.A., Keane, J.F. and Lock, J.E. (1998) Aortic Valve-Preserving Procedure for Enlargement of the Left Ventricular Outflow Tract and Mitral Anulus. The Journal of Thoracic and Cardiovascular Surgery, 115, 1219-1222. http://dx.doi.org/10.1016/S0022-5223(98)70427-1 
Scientific Research Publishing (SCIRP) is one of the largest Open Access journal publishers. It is currently publishing more than 200 open access, online, peer-reviewed journals covering a wide range of academic disciplines. SCIRP serves the worldwide academic communities and contributes to the progress and application of science with its publication.

Other selected journals from SCIRP are listed as below. Submit your manuscript to us via either submit@scirp.org or Online Submission Portal.
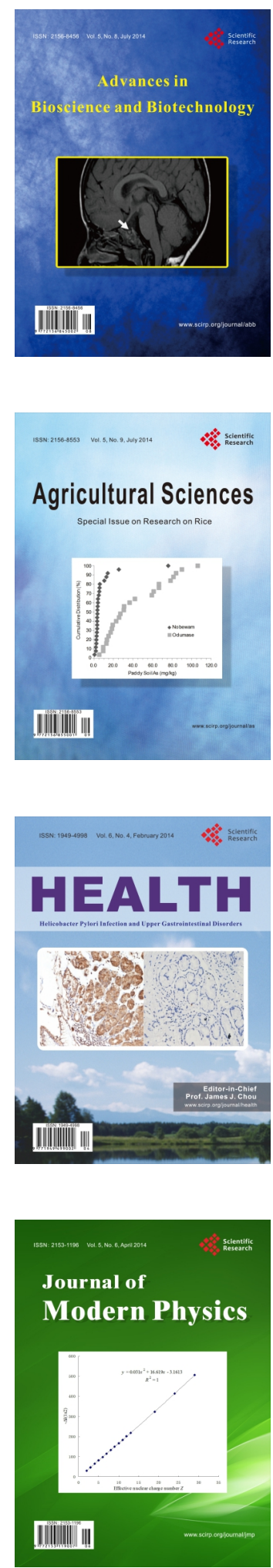
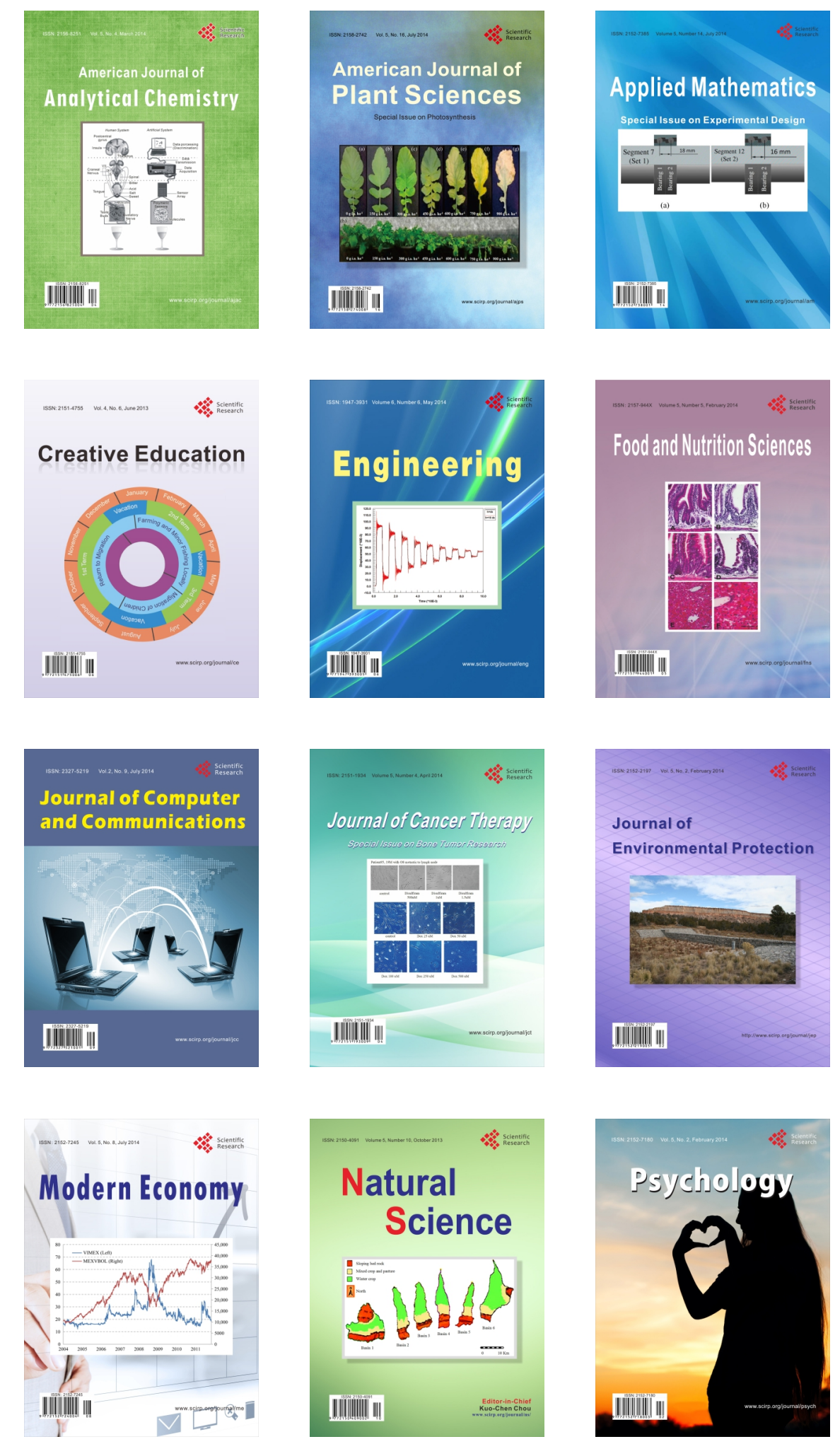